\title{
Thyroid Transcription Factor-1 Expression in Adenocarcinoma Lung and Its Association with Histomorphological Features
}

\begin{abstract}
Objectives: Thyroid transcription factor-1 (TTF-1) expression is frequently associated with adenocarcinoma lung. This study was undertaken with the objectives to assess the expression of TTF-1 in non-small cell lung carcinoma (NSCLC) with adenocarcinoma phenotype and to evaluate the TTF-1 expression with clinicopathological and histomorphological features. Materials and Methods: This was a tertiary care hospital-based case series that included 250 cases of NSCLC. TTF-1 immunostaining along with a basic panel of immunohistochemistry markers was performed. The histomorphological analysis was done to compare the morphological features of TTF-1-positive versus TTF-1-negative cases. Results: TTF-1 was positive in 144 cases (57.6\%), while 106 cases were negative for TTF-1. The M: F ratio in the TTF-1-positive group was 1:2, and the mean age of the cases that expressed TTF-1 was 48.5 years. The most common pattern in the TTF-1-positive group was loose clusters or singly dispersed cells $(77.78 \%)$, followed by the acinar $(54.86 \%)$ and solid pattern $(42.36 \%)$. In the TTF-1-negative group, $86.79 \%$ of cases had singly dispersed cells or loose cohesive clusters, followed by a solid pattern in $50.94 \%$ cases. Conclusion: TTF-1 is a useful and reliable marker for pulmonary adenocarcinoma. TTF-1 expression does not have any association with the patterns and degree of differentiation evident in the tumor.
\end{abstract}

Keywords: Adenocarcinoma, lung, morphological patterns, thyroid transcription factor-1, tumor differentiation

\section{Introduction}

The main cause of cancer-related deaths, in the era of modern medicine, is lung cancer. Despite the immense advances in the therapeutics and the advent of personalized medicine, the overall survival is poor. ${ }^{[1,2]}$ Lung cancer is broadly divided into two categories for the tenacities of diagnosis and treatment: small cell lung carcinoma (SCLC) and non-SCLC (NSCLC), the latter consisting of adenocarcinoma, squamous cell carcinoma, neuroendocrine tumors, sarcomatoid carcinoma, and undifferentiated carcinomas. SCLC constitutes approximately $15 \%$ of lung cancers, has a very poor prognosis and generally presents at an advanced stage with metastatic disease. The main modality of the treatment for SCLC is chemoradiation. NSCLC constitutes nearly $85 \%$ of lung cancers, and adenocarcinoma is the major subtype of this category. ${ }^{[2-4]}$ Targeted therapy now forms the mainstay of treatment in cases of NSCLC with

This is an open access journal, and articles are distributed under the terms of the Creative Commons Attribution-NonCommercial-ShareAlike 4.0 License, which allows others to remix, tweak, and build upon the work non-commercially, as long as appropriate credit is given and the new creations are licensed under the identical terms.

For reprints contact: reprints@medknow.com adenocarcinoma phenotype along with immunotherapy. ${ }^{[5]}$

Thyroid transcription factor-1 (TTF-1) belongs to the family transcription factors. TTF-1 plays a major role in the initiation of specific genes in brain, thyroid, and lung. ${ }^{[6]}$ Differentiation and embryonic development are also governed by TTF-1. TTF-1 is frequently expressed in NSCLC-adenocarcinoma phenotype. In the normal lung, TTF-1 expression is limited to the Type II alveolar cells. Despite numerous studies, the factors that control TTF-1 expression associated with differentiation and prognostic implications have not been completely documented. ${ }^{[7,8]}$

This study was undertaken with the objectives to assess the expression of TTF-1 in NSCLC with adenocarcinoma phenotype and to evaluate the TTF-1 expression with clinicopathological and histomorphological features.

\section{Materials and Methods}

The current study was a tertiary care hospital-based case series of 250 cases

\footnotetext{
How to cite this article: Shukla S, Husain N, Anand N, Kant S, Garg R, Verma SK, et al. Thyroid transcription factor-1 expression in adenocarcinoma lung and its association with histomorphological features. Indian J Med Paediatr Oncol 2019;40:510-4.
}

\section{Saumya Shukla', Nuzhat Husain ${ }^{1}$, Nidhi Anand', Surya Kant ${ }^{2}$, Rajiv Garg", SK Verma ${ }^{2}$, Santosh Kumar', Ved Prakash ${ }^{2}$}

${ }^{1}$ Department of Pathology, Dr. Ram Manohar Lohia Institute of Medical Sciences, ${ }^{2}$ Department of Respiratory Medicine, King George's Medical University, Lucknow, Uttar Pradesh, India

Submitted: 11-Sep-2018 Revised: 04-Apr-2019 Accepted: 04-Aug-2019 Published: 17-Feb-2020

Address for correspondence: Dr. Saumya Shukla, Department of Pathology, Dr. Ram Manohar Lohia Institute of Medical Sciences, Lucknow, Uttar Pradesh, India. E-mail: saumyavarun@gmail. com Website: www.ijmpo.org DOI: 10.4103/ijmpo.ijmpo_199_18 Quick Response Code:

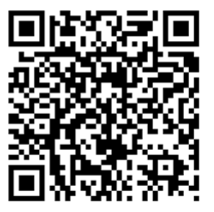


of NSCLC. Adequate clinical and radiological details were obtained in all cases. The study included biopsies from both primary and metastatic sites of NSCLC adenocarcinoma, adenosquamous carcinoma, excluding squamous cell carcinoma phenotype. All the cases were categorized histopathologically as per the 2015 World Health Organization classification of lung tumors. An elaborate panel of immunohistochemistry (IHC) used for the classification of the cases [Table 1].

\section{Protocol for immunohistochemistry}

IHC was performed on formalin-fixed paraffin-embedded (FFPE) tissue blocks. The FFPE tissue blocks were sectioned using a microtome (Leica, Germany) at a thickness of 3-4 $\mu \mathrm{m}$ and mounted on glass slides coated with tissue bond from Biocare, USA. These coated slides with the tissue sections were fixed overnight in an oven at $60^{\circ} \mathrm{C}$. Xylene was used for deparaffinization followed by rehydration using a series of graded alcohol. Blocking was done to quench the endogenous peroxidize activity using 3\% hydrogen peroxide in methanol for $30 \mathrm{~min}$. This was followed by antigen retrieval using the sodium citrate buffer ( $\mathrm{pH}-6.0)$ done in Pascal from DAKO Cytomation, California, USA. The sections were incubated with the primary antibody. All the primary antibodies (including TTF-1) were incubated for 1 hour. The Envision secondary antibody used was from Dakopatts Denmark was used. The visualization was done as per the manufacturer's instructions using diaminobenzidine. All the sections were adequately counterstained with hematoxylin and mounted for light microscopy visualization. All the slides were run in batches, including positive and negative controls.

The positive control used for TTF-1 IHC staining was normal thyroid tissue while negative controls were tissue in which the primary antibody application had been omitted.

The assessment of thyroid transcription factor-1 immunostaining

The assessment of immunostaining was done using light microscopy. The tumors were categorized as positive and negative by assessing nuclear staining for TTF-1. Tumors

\begin{tabular}{llll}
\hline \multicolumn{4}{c}{ Table 1: List of primary immunohistochemistry } \\
antibodies used for accurate & characterization cases \\
\hline Name & Clone & Supplier & Dilution \\
\hline CK7 & OV-TL 12/30 & Dako & Ready to use \\
CK20 & Ks20.8 & Dako & Ready to use \\
TTF-1 & 8G7G3/1 & Dako & Ready to use \\
CDX-2 & DAK-CDX2 & Dako & Ready to use \\
Thyroglobulin & DAK-Tg6 & Dako & $1: 100$ \\
Napsin-A & TMU-Ad02 & Biocare & Ready to use \\
PAX-8 & BC-12 & Biocare & Ready to use \\
p-40 & p-40 (M) & Biocare & Ready to use \\
\hline CK 7 - Cytokeratin 7; CK20-Cytokeratin 20; TTF-1 - Thyroid \\
Transcription Factor-1
\end{tabular}

were considered positive when $>5 \%$ cells harbored appreciable nuclear staining. Any tumor with lack of perceptible nuclear staining was categorized as negative.

\section{Histomorphological analysis}

A detailed histomorphological analysis was performed for all cases. The analysis included the identification of various patterns of tumor cell arrangement namely solid, micropapillary, lepidic, acinar, and loose clusters/ singly dispersed cells along with the presence of mucin (intracytoplasmic/extracytoplasmic). In addition, the presence/absence of necrosis was documented in all the cases. All the tumors were graded based on the histomorphology into well, moderately, and poorly differentiated tumors.

\section{Statistical analysis}

The IBM-Statistical Package for Social Sciences (SPSS, International Business Machines Corporation., New York, USA) analysis software, version 16 was used for all statistical calculations. The categorical variables were compared using the Chi-square test. Two-sided tests were used for the calculation of all $P$ values, and $P \leq 0.05$ was considered statistically significant, whereas $P \leq 0.01$ was considered highly significant. The histomorphology of the TTF-1-positive cases versus the TTF-1-negative cases was compared.

\section{Results}

This was a tertiary care hospital-based case series that included 250 cases of NSCLC. Biopsies from both primary and metastatic sites of NSCLC adenocarcinoma, adenosquamous carcinoma excluding the squamous cell carcinoma subtype were included in the study.

The age range of the cases varied from 22 to 86 years with a mean age of 56.81 years, with $35.48 \%$ cases between the age range of 51 and 60 years.

The male to female ratio was $2: 1$.

The site of the biopsy was from the respiratory tree (endobronchial, intrathoracic, lung, or pleural) in $88.5 \%$ cases, while biopsy was performed from the metastatic sites in $11.5 \%$ cases. In cases of metastatic lesions, the primary lesion was diagnosed in the lung with the aid of IHC. Among the metastatic sites, the most common was lymph node $(41.27 \%)$.

The most common clinical finding in the cases was the presence of lung mass with or without the presence of pleural effusion.

Napsin-A and cytokeratin (CK)-7 were positive in all 250 cases. In biopsies that were performed from the respiratory tree, TTF-1 was positive in 115 cases. In all the cases, co-expression of napsin-A, CK-7, and TTF-1 aided in diagnosis of NSCLC-adenocarcinoma.

TTF-1 was negative in 106 cases. All these biopsies were from the respiratory tree. All these 106 cases had a positive 
expression of CK-7 and napsin-A. However, the additional panel of markers including PAX-8 and thyroglobulin was run. All 106 cases were negative for both these markers, following which a diagnosis of NSCLC-adenocarcinoma was rendered.

In this cases series, 29 biopsies were from metastatic sites. The panel used included CK-7, CK20, TTF-1, napsin-A, thyroglobulin, PAX-8, and CDX-2. In biopsies from metastatic sites, CK-7, napsin-A, and TTF-1 were positive in all 29 cases, while the other markers were negative. Based on these results, the diagnosis of metastatic pulmonary adenocarcinoma was rendered.

TTF-1 was positive in 144 cases (57.6\%), whereas 106 cases were negative for TTF-1 [Table 2].

Markers for squamous differentiation were performed in all 250 cases, and squamous differentiated was identified in three cases (1.2\%) [Figures 1 and 2].

Characteristics of thyroid transcription factor-1-positive cases

The M:F ratio in the TTF-1-positive group was 1:2 and the mean age of the cases that expressed TTF-1 was 48.5 years.

The histomorphological features of the TTF-1 positive $(n=144)$ and the TTF-1 negative $(n=106)$ were analyzed. Most of the cases had a mixed pattern on histomorphological analysis. The most common pattern in the TTF-1-positive group was loose clusters or singly dispersed cells $(77.78 \%)$, followed by the acinar $(54.86 \%)$ and solid pattern $(42.36 \%)$. Necrosis was present in $47.22 \%$ cases, and $50 \%$ of the cases were moderately differentiated with a nuclear Grade 2 in $64.58 \%$ cases.

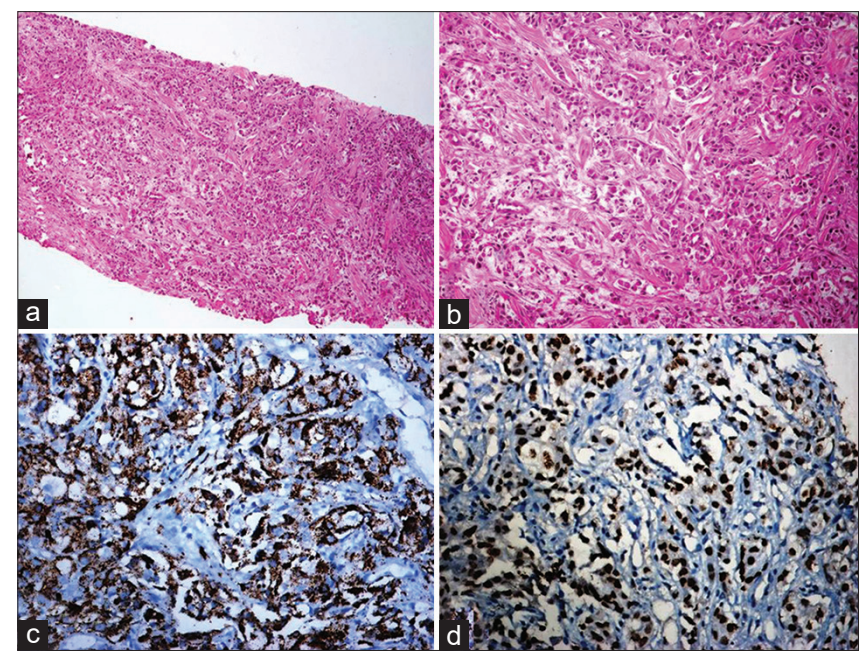

Figure 1: Guided biopsy from an intrathoracic mass: ( $a$ and $b$ ): A tumour composed of cells arranged in an acinar pattern. The tumour cells have high nucleo-cytoplasmic ratio, moderate amount of cytoplasm, ploemorphic vesicular nucleus with prominent nucleoli $[a=H$ and $E \times 100, b=H$ and $E$ $\times 200]$ (c): Napsin-A: Positive [DAB ×100], (d): TTF 1: Positive [DAB ×100]. $H$ and $E=H e m a t o x y l i n$ and Eosin, DAB=Diaminobenzidin
In the TTF-1-negative group, $86.79 \%$ of cases had singly dispersed cells or loose cohesive clusters, followed by a solid pattern in $50.94 \%$ cases. Necrosis was present in $55.66 \%$ of cases. However, none of these parameters between the TTF-1 positive and the TTF-1-negative group was statistically significant [Table 3 and Figure 3].

\section{Discussion}

TTF-1 is a nuclear protein-related transcription factor, localized in chromosome $14 \mathrm{q} 13$ and is present in approximately $75 \%$ of lung adenocarcinomas. ${ }^{[7]}$ During growth and embryogenesis, TTF-1 expression is limited to respiratory epithelium, diencephalon of the brain and the thyroid. TTF-1 plays a pivotal role as the chief regulatory factor in lung development. The alternative major function of TTF-1 includes the activation of factors for

\begin{tabular}{|c|c|c|}
\hline \multirow{3}{*}{ IHC marker } & \multirow{2}{*}{\multicolumn{2}{|c|}{ Number of cases }} \\
\hline & & \\
\hline & $\begin{array}{l}\text { Positive } \\
\text { staining }\end{array}$ & $\begin{array}{l}\text { Negative } \\
\text { staining }\end{array}$ \\
\hline Napsin-A (performed in 250 cases) & 250 & 0 \\
\hline CK7 (performed in 250 cases) & 250 & 0 \\
\hline TTF-1 (performed in 250 cases) & 144 & 106 \\
\hline $\begin{array}{l}\text { Markers of squamous differentiation (p40) } \\
\text { (performed in } 250 \text { cases) }\end{array}$ & 3 & 247 \\
\hline CK20 (performed in 29 cases) & 0 & 29 \\
\hline CDX-2 (performed in 29 cases) & 0 & 29 \\
\hline Thyroglobulin (performed in 135 cases) & 0 & 135 \\
\hline PAX-8 (performed in 135 cases) & 0 & 135 \\
\hline
\end{tabular}

IHC - Immunohistochemistry; CK7 - Cytokeratin 7;

CK20 - Cytokeratin 20; TTF-1 - Thyroid Transcription Factor-1

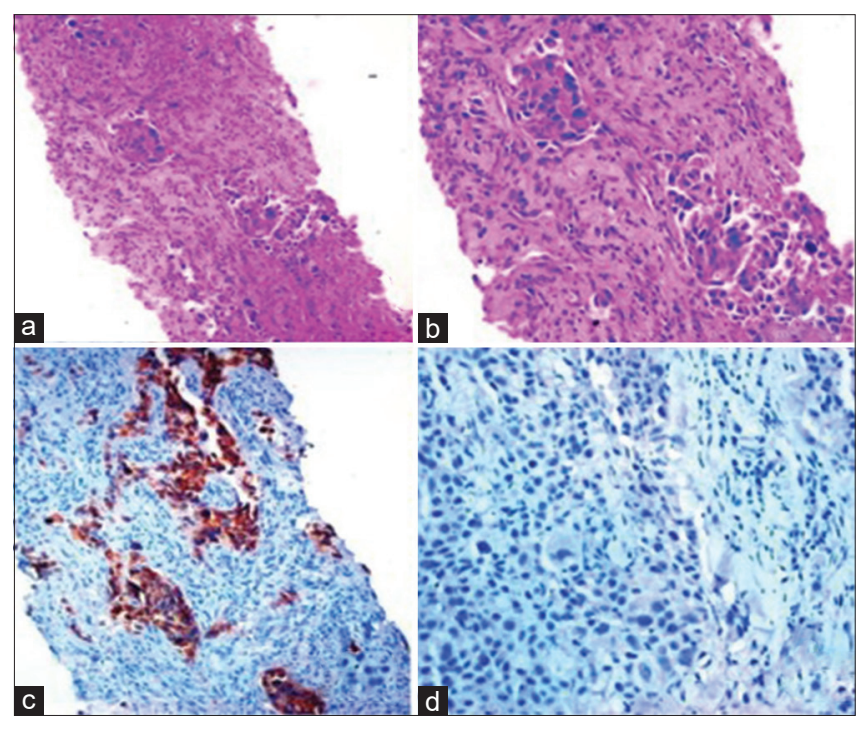

Figure 2: Thyroid transcription factor-1-negative adenocarcinoma. Guided biopsy from lung mass: (a and b) Lung parenchyma infiltrated by a tumour with solid and acinar pattern ([a] $H$ and $E, \times 100,[b] H$ and E, $\times 200)(c)$ Napsin-A: Positive (DAB $\times 100)$, (d) Thyroid transcription factor-1: Negative (DAB $\times 200$ ). $H$ and $E=H e m a t o x y l i n$ and Eosin, $\mathrm{DAB}=$ Diaminobenzidine 


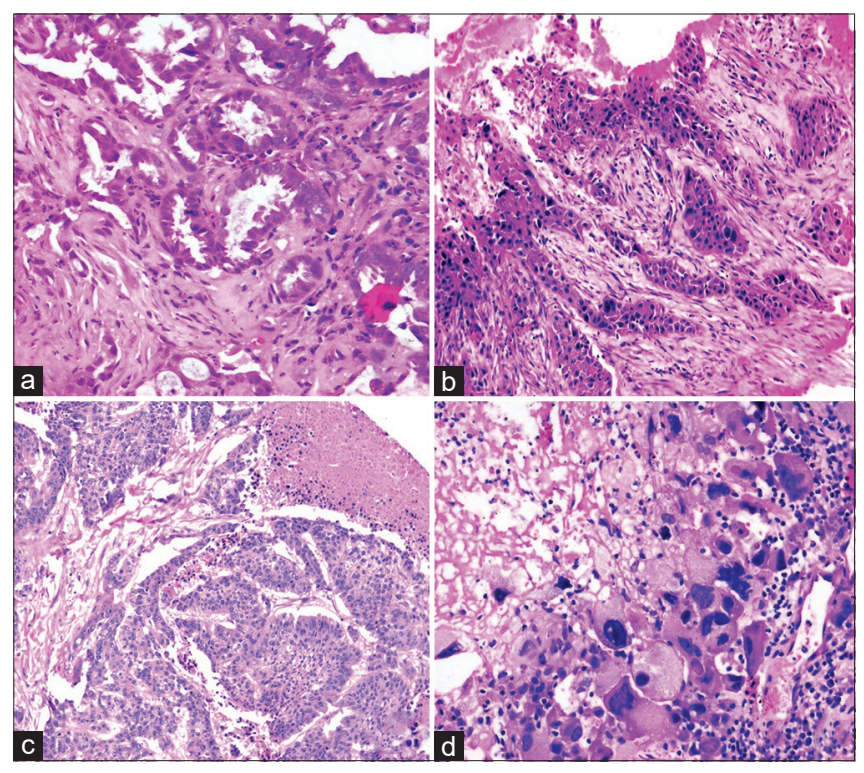

Figure 3: Morphological pattern: (a) Acinar Pattern (b) solid nests with cords, (c) micropapillary pattern with necrosis, (d) poorly differentiated morphology with high nuclear grade ([a-d] $\mathrm{H}$ and $\mathrm{E}, \times 100)$. $\mathrm{H}$ and $\mathrm{E}=$ Hematoxylin and Eosin

the production of surfactant protein released by the Clara cells. The function of the terminal respiratory unit (TRU) is essentially maintained by TTF-1. ${ }^{[9,10]}$ The inactivation of TTF-1 has been described to cause tracheoesophageal fistula, pulmonary branching disorders, and pulmonary hypoplasia. ${ }^{[11]}$

In the current study, TTF-1 expression was identified in $57.6 \%$ cases which is lower than the average percentage described in literature. This can partly be explained by the fact that the majority of tumors in this case series were either moderately or poorly differentiated $(n=229)$. TTF-1 expression is generally lost in larger high-grade tumors associated with advanced disease. ${ }^{[1]}$

In the present study, TTF-1 expression was more common in females. This is in concordance with the studies conducted by Yatabe et al. and Zhang et al. ${ }^{[7,11]}$

The average age of the cases that expressed TTF-1 protein in the present study was 48.5 years which is lower than the overall average age of the study group in this case series. This finding may indicate the TTF-1 expression is common in adenocarcinomas in the young population. ${ }^{[12,13]}$

In the present study, the assessment of morphological patterns of TTF-1-positive versus TTF-1-negative cases revealed that in both the groups, the tumor cells were predominantly disposed in loose clusters or were singly scattered. However, the most common pattern in the TTF-1-positive group was the acinar pattern $(54.86 \%)$ while the solid pattern dominated in the TTF-1-negative group (50.94\%). In the study conducted by Zhou et al., the acinar pattern was predominant in both the TTF-1-positive and TTF-1-negative groups. ${ }^{[14]}$ The findings of the study conducted by Stenhouse et al., are in concordance with the results of the current study with

\begin{tabular}{|c|c|c|c|}
\hline \multicolumn{4}{|c|}{$\begin{array}{l}\text { Table 3: Significance of association of histomorpholo } \\
\text { characteristics in Thyroid Transcription } \\
\text { Factor-1-positive versus Thyroid Transcription } \\
\text { Factor-1-negative cases }\end{array}$} \\
\hline Morphological characteristics & TTF-1 + (\%) & TTF-1 - (\%) & $P$ \\
\hline \multicolumn{4}{|l|}{ Solid pattern } \\
\hline Present & $61(42.36)$ & $54(50.94)$ & 0.18 \\
\hline Absent & $83(57.64)$ & $52(49.06)$ & \\
\hline \multicolumn{4}{|l|}{ Micropapillary pattern } \\
\hline Present & $26(18.06)$ & $5(4.72)$ & 0.32 \\
\hline Absent & $118(81.94)$ & $101(95.28)$ & \\
\hline \multicolumn{4}{|l|}{ Acinar pattern } \\
\hline Present & $79(54.86)$ & $50(47.17)$ & 0.35 \\
\hline Absent & $65(45.14)$ & $56(52.83)$ & \\
\hline \multicolumn{4}{|l|}{ Lepidic pattern } \\
\hline Present & $5(3.47)$ & $7(6.60)$ & 0.25 \\
\hline Absent & $139(96.53)$ & $99(93.4)$ & \\
\hline \multicolumn{4}{|l|}{$\begin{array}{l}\text { Mucin (intracytoplasmic/ } \\
\text { extracytoplasmic) }\end{array}$} \\
\hline Present & $44(30.56)$ & $23(21.69)$ & 0.12 \\
\hline Absent & $100(69.44)$ & $83(78.31)$ & \\
\hline \multicolumn{4}{|l|}{ Loose clusters/dispersed cells } \\
\hline Present & $112(77.78)$ & $92(86.79)$ & 0.07 \\
\hline Absent & $32(22.22)$ & $14(13.21)$ & \\
\hline \multicolumn{4}{|l|}{ Necrosis } \\
\hline Present & $68(47.22)$ & $59(55.66)$ & 0.19 \\
\hline Absent & $76(52.78)$ & $47(44.34)$ & \\
\hline \multicolumn{4}{|l|}{ Differentiation } \\
\hline Well & $15(10.42)$ & $6(5.66)$ & 0.39 \\
\hline Moderately & $72(50)$ & $54(50.94)$ & \\
\hline Poorly & $57(39.58)$ & $46(43.39)$ & \\
\hline \multicolumn{4}{|l|}{ Nuclear grade } \\
\hline Grade 1 & $17(11.81)$ & $8(7.54)$ & 0.23 \\
\hline Grade 2 & $93(64.58)$ & $79(29.81)$ & \\
\hline Grade 3 & $34(23.61)$ & $19(17.92)$ & \\
\hline
\end{tabular}

TTF-1 - Thyroid Transcription Factor-1

a predominance of the solid pattern in the TTF-1-negative group. ${ }^{[10]}$ A mucinous component was identified in $30.56 \%$ of cases with TTF-1 expression. This feature is contrary to the conclusions of Zhang et al., Stenhouse et al., Zhou et al., and Yaman et al..$^{[10,11,14,15]}$

In the present study, necrosis was a predominant feature of the TTF-1-negative group and was present in $55.66 \%$ cases while $94.33 \%$ of cases were either moderately or poorly differentiated tumors. A reciprocal correlation between tumor differentiation and TTF-1 expression has been described by Huang et al. ${ }^{[16]}$ Yaman et al. concluded from their study that poorly differentiated tumors were generally TTF-1 negative. ${ }^{[15]}$

The staining pattern of TTF-1 is largely uniform in the adenocarcinomas in spite of histologic and microenvironmental multiplicity in individual tumors. This consistency and homogeneity imply that TTF-1 expression could be used as a lineage marker of TRU. ${ }^{[7,17]}$ 
Several studies have been conducted to evaluate the association between TTF-1 expression and prognosis. TTF-1 loss is principally associated with larger tumors with advanced clinically stage and aggressive course. ${ }^{[14,16,18]}$

In the study conducted by Zhou et al. no statistical variance was obtained in the overall survival of cases that were TTF-1 versus the TTF-1-negative cases. Stenhouse et al. in their study stated that the absence of TTF-1 staining was not related to tumor prognosis or survival. ${ }^{[10,14]}$

\section{Conclusion}

TTF-1 is a useful and consistent marker for pulmonary adenocarcinoma. The analysis of the histomorphological characteristics revealed that TTF-1 expression has no association with the patterns and degree of differentiation evident in the tumor.

\section{Financial support and sponsorship}

Nil.

\section{Conflicts of interest}

There are no conflicts of interest.

\section{References}

1. Jemal A, Bray F, Center MM, Ferlay J, Ward E, Forman D. Global cancer statistics. CA Cancer J Clin 2011;61:69-90.

2. Devesa SS, Bray F, Vizcaino AP, Parkin DM. International lung cancer trends by histologic type: Male: Female differences diminishing and adenocarcinoma rates rising. Int $\mathrm{J}$ Cancer 2005;117:294-9.

3. Kaufmann O, Dietel M. Thyroid transcription factor-1 is the superior immunohistochemical marker for pulmonary adenocarcinomas and large cell carcinomas compared to surfactant proteins A and B. Histopathology 2000;36:8-16.

4. Chang YL, Lee YC, Liao WY, Wu CT. The utility and limitation of thyroid transcription factor-1 protein in primary and metastatic pulmonary neoplasms. Lung Cancer 2004;44:149-57.

5. Dietel M, Bubendorf L, Dingemans AM, Dooms C, Elmberger G, García RC, et al. Diagnostic procedures for non-small-cell lung cancer (NSCLC): Recommendations of the European expert group. Thorax 2016;71:177-84.

6. Inamura $\mathrm{K}$, Takeuchi $\mathrm{K}$, Togashi $\mathrm{Y}$, Hatano S, Ninomiya $\mathrm{H}$,
Motoi N, et al. EML4-ALK lung cancers are characterized by rare other mutations, a TTF-1 cell lineage, an acinar histology, and young onset. Mod Pathol 2009;22:508-15.

7. Yatabe Y, Mitsudomi T, Takahashi T. TTF-1 expression in pulmonary adenocarcinomas. Am J Surg Pathol 2002;26:767-73.

8. Zamecnik J, Kodet R. Value of thyroid transcription factor-1 and surfactant apoprotein $\mathrm{A}$ in the differential diagnosis of pulmonary carcinomas: A study of 109 cases. Virchows Arch 2002;440:353-61.

9. Tanaka H, Yanagisawa K, Shinjo K, Taguchi A, Maeno K, Tomida $\mathrm{S}$, et al. Lineage-specific dependency of lung adenocarcinomas on the lung development regulator TTF-1. Cancer Res 2007;67:6007-11.

10. Stenhouse G, Fyfe N, King G, Chapman A, Kerr KM. Thyroid transcription factor 1 in pulmonary adenocarcinoma. J Clin Pathol 2004;57:383-7.

11. Zhang $\mathrm{Y}$, Wang $\mathrm{R}$, Li $\mathrm{Y}$, Pan $\mathrm{Y}, \mathrm{Hu} \mathrm{H}$, Zhang $\mathrm{Y}$, et al. Negative thyroid transcription factor 1 expression defines an unfavorable subgroup of lung adenocarcinomas. J Thorac Oncol 2015; 10:1444-50.

12. Li X, Wan L, Shen H, Geng J, Nie J, Wang G, et al. Thyroid transcription factor-1 amplification and expressions in lung adenocarcinoma tissues and pleural effusions predict patient survival and prognosis. J Thorac Oncol 2012;7:76-84.

13. Anagnostou VK, Syrigos KN, Bepler G, Homer RJ, Rimm DL. Thyroid transcription factor 1 is an independent prognostic factor for patients with stage I lung adenocarcinoma. J Clin Oncol 2009;27:271-8.

14. Zhou C, Zhao J, Shao J, Li W. Prognostic relevance of TTF-1 expression in stage I adenocarcinoma. Oncotarget 2017;8:107462-8.

15. Yaman B, Nart D, Ekren PK, Çok G, Veral A. Expression of p63, TTF-1 and maspin in non-small cell lung carcinoma and their effect on the prognosis and differential diagnosis. Turk Patoloji Derg 2015;31:163-74.

16. Huang TW, Lin KF, Lee CH, Chang H, Lee SC, Shieh YS. The role of thyroid transcription factor-1 and tumor differentiation in resected lung adenocarcinoma. Sci Rep 2017;7:14222.

17. Solis LM, Behrens C, Raso MG, Lin HY, Kadara H, Yuan P, et al. Histologic patterns and molecular characteristics of lung adenocarcinoma associated with clinical outcome. Cancer 2012;118:2889-99.

18. Barletta JA, Perner S, Iafrate AJ, Yeap BY, Weir BA, Johnson LA. Clinical significance of TTF-1 protein expression and TTF-1 gene amplification in lung adenocarcinoma. J Cell Mol Med 2009;13:1977-86. 